\title{
Performance of a dipstick dye immunoassay for rapid screening of Schistosoma japonicum infection in areas of low endemicity
}

Jing Xu1, Ting Feng ${ }^{1}$, Dan-Dan Lin², Qi-Zhi Wang ${ }^{3}$, Li Tang $^{4}$, Xiao-Hua Wu' ${ }^{1}$ Jia-Gang Guo ${ }^{1}$, Rosanna W Peeling ${ }^{5}$ and Xiao-Nong Zhou ${ }^{1 *}$

\begin{abstract}
Background: The dipstick dye immunoassay (DDIA), recently commercially available in the People's Republic of China (P.R. China), is a rapid and simple test to detect human antibodies against Schistosoma Japonicum. Its performance and utility for screening schistosome infection in low endemic areas is little known. We therefore carried out a cross-sectional survey in seven villages with low endemicity of schistosomiasis in P.R. China and assessed the performance and utility of DDIA for diagnosis of schistosomiasis. Stool samples were collected and examined by the Kato-Katz method and the miracidium hatching technique. Serum samples, separated from whole blood of participants, were tested by DDIA.

Results: 6285 individuals aged 6-65 years old participated in this study, with a prevalence of schistosomiasis of 4.20\%. Using stool examination as a gold reference standard, DDIA performed with a high overall sensitivity of 91.29\% (95\% Cl: 87.89-94.69\%) and also a high negative predictive value, with a mean value of $99.29 \%$ (95\% Cl: 98.99-99.58\%). The specificity of DDIA was only moderate (53.08\%, 95\% Cl: $51.82-54.34 \%)$. Multivariate analysis indicated that age, occupation and history of schistosome infection were significantly associated with the false positive results of DDIA.
\end{abstract}

Conclusions: DDIA is a sensitive, rapid, simple and portable diagnostic assay and can be used as a primary approach for screening schistosome infection in areas of low endemicity. However, more sensitive and specific confirmatory assays need to be developed and combined with DDIA for targeting chemotherapy accurately.

\section{Background}

Schistosomiasis japonica is one serious infectious disease, draining the economic and social development in the People's Republic of China (P.R. China) [1]. An estimated 100 million people were at risk of contracting schistosomiasis and 11.6 million were infected in 12 endemic provinces in P.R. China in the mid $20^{\text {th }}$ century $[1,2]$. With continuous national programs being implemented in P.R. China, great achievements have been made in the control of schistosomiasis. The prevalence and intensity of Schistosoma japonicum (S. japonicum) infection have decreased dramatically. Most counties

\footnotetext{
* Correspondence: ipdzhouxn@sh163.net

'National Institute of Parasitic Diseases, Chinese Center for Disease Control and Prevention, WHO Collaborating Center for Malaria, Schistosomiasis and Filariasis, Shanghai 200025, People's Republic of China

Full list of author information is available at the end of the article
}

have reached the criteria of infection control (human prevalence less than $5 \%$ ), while in many others, transmission control (human prevalence less than $1 \%$ ) or even transmission interruption (no case found in five consecutive years) has been achieved [3]. These different endemic levels increase the demand of sensitive and cost-effective diagnosis for accurate identification of schistosomiasis cases, followed by treatment of individuals and/or communities, and evaluation of intervention efficacy as the control goal is still to reduce the prevalence to a sustainable low level [4].

Due to lack of other pragmatic diagnostic methods, the Kato-Katz method is still the most widely used for direct diagnosis of intestinal schistosomiasis in P.R. China, although it fails due to its insensitivity in regions of low endemicity and light infections, especially when only one stool specimen is used for diagnosis $[5,6]$.

\section{Biomed Central}


Combination of the Kato-Katz method and the miracidium hatching technique could decrease the misdiagnosis of patients, but the performance of the latter is prone to be affected by various factors such as temperature and quality of water [7-10]. Furthermore, direct stool examinations on a population level to find a few cases will be costly and are not appropriate in areas of low endemicity. And the compliance of residents to provide stool specimens were also decreased year after year $[11,12]$. To overcome these shortcomings, a two-step method has been implemented for guiding chemotherapy, estimation of endemic status, and assessment of intervention efficiency in the schistosomiasis control programs in P.R. China, with antibody-based immunoassay as a primary approach for screening the human population due to its higher sensitivity and simple operational characteristics. Only antibody positive cases are followed by stool examination to make sure whether they are currently infected with schistosomes. [2,13-16].

Facilitated and improved by advances in immunolabeling techniques, there are several kinds of immunoassays for diagnosis of schistosome infection which have been developed and implemented for screening, such as the circumoval precipitin test (COPT), indirect hemagglutination test (IHA), enzyme-linked immunosorbent assay (ELISA), etc. $[13,17,18]$. But the intrinsic features of these assays, such as complex and time-consuming procedure, needs of extra instruments etc., have limited their use on a large scale in field settings especially in areas of low endemicity with limited resources [19]. There is an increased need for sensitive, rapid, simple and inexpensive assays for screening of schistosomiasis, especially in the case of on-the-spot surveys in low endemicity areas.

With the growing interest in the use of rapid diagnostic test for schistosome infection, dipsticks, based on lateral immunochromatographic flow method, have been used to detect circulating cathodic antigen (CCA) of Schistosoma mansoni infection and proved to be an alternative methodology for estimating infection prevalence and intensity [20]. Recently, a rapid and simple test named dipstick dye immunoassay (DDIA) has been made commercially available in P.R. China market to detect human antibodies against schistosomes. This assay can be done in 5-10 minutes per test without additional equipment except a micropipettor [21]. Laboratory-based evaluation and field trials proved that DDIA performed with high sensitivity in areas with high endemicity and high specificity in areas free of schistosome infection $[22,23]$. In this study, we report on the performance of DDIA compared with stool examination and evaluate its efficacy as a primary approach for screening the population in seven villages of low endemicity.

\section{Methods}

\section{Study areas, population and sample collection}

A cross-sectional survey was carried out in seven separate administrative villages from three provinces along Yangtz River in China: Caohui, Jingtou and Xinhua villages located in Jiangxi Province, Longshang, Tieguai and Yuye villages from Anhui province, and Hebei village administrated by Hubei province. We invited all individuals aged from 6-65 years old in each village to participate in our study which was performed in October and November 2008.

Demographic data of individuals, including age, gender, occupation, birth date and history of schistosome infection and other related diseases, were recorded by a structured questionnaire. Each participant was asked to provide one stool sample over $50 \mathrm{~g}$ and one blood sample over $250 \mu \mathrm{l}$ through finger prick. The samples were taken to local schistosomiasis stations, where stool examinations and DDIA tests were performed by laboratory staff.

\section{Ethical statement}

Written informed consent was obtained from all adult participants and from the parents or legal guardians of children. The study was approved by the ethical committee of National Institute of Parasitic Diseases, China Center for Disease Control and Prevention. Any participant found to be parasitologically positive received anthelmintic treatment except for those with contraindications.

\section{Stool examination}

Each stool specimen was processed using the Kato-Katz thick smear method and the miracidium hatching technique without knowledge of the infection result of previous diagnostics.

Kato-Katz technique was performed as previously described to detect $S$. japonicum eggs in stool samples [24]. Briefly, three slides from a single stool specimen were prepared using a standard template $(41.7 \mathrm{mg}$ per smear). All slides were read $12-48 \mathrm{~h}$ after their initial preparation by two qualified technicians, who were unaware of the subjects' medical status, results of miracidium hatching technique and DDIA detection. The number of eggs in each slide was counted and recorded. Infection intensity of patients was expressed as eggs per gram of feces (EPG), using arithmetic mean of all eggs counts obtained from three slides multiplied by 24 .

Miracidium hatching technique was performed as follows: $30 \mathrm{~g}$ of feces were homogenized in dechlorinated water and then filtered over double-layered gauze to remove large detritus, repeating the filtration until a clear supernatant liquid was obtained. The suspension was then transferred to a volumetric flask and hatched in $26-30^{\circ} \mathrm{C}$ in an illumination incubator. Observations 
were taken 4,8 and 12 hours after hatching with the naked eye or by means of a magnifying glass. The result was defined as positive if moving miracidia in the supernatant were observed [25]. The infection intensity of cases that were considered negative by the Kato-Katz method but positive by the miracidium hatching technique was regarded as one EPG.

Combining the results of the Kato-Katz method and the miracidium hatching technique, a patient was defined as positive if positive results were obtained with either parasitological technique and as negative if negative results were obtained with both parasitological methods. The infection intensity of cases was divided into three categories as $S$. mansoni defined by World Health Organization: light infection (1-99 EPG), moderate infection (100-399 EPG) and heavy infection ( $\geq 400$ EPG) [26].

\section{DDIA}

Serum was separated from the whole blood sample and was tested, without prior knowledge of the results of stool examination. DDIA kits were supplied by Wuxi Saide Medical Technology Co. Ltd, Jiangsu, P.R. China (Product Lot: 0809101). The test uses soluble antigen extracted from $S$. japonicum eggs as the probe to detect antibodies against $S$. japonicum in human blood [21].

Serological tests were performed according to the instructions supplied by the manufacturer. Briefly, a drop of $50 \mu \mathrm{l}$ blue colloidal dye-labeled soluble egg antigens (SEA) solution from the buffer bottle was added into a polyvinyl chloride (PVC) well and $20 \mu \mathrm{l}$ of serum specimen was added. The solution in the well was mixed lightly for about one minute. A dipstick was then inserted into the well. The result was read after the solution was absorbed completely. The appearance of two blue bands on the dipstick indicated a positive reaction, and the appearance of a single blue band in the control position indicated a negative reaction [21].

\section{Statistical analysis}

In the field survey, only data from subjects who accepted stool examination and serological tests were used for the analysis. The combination of the Kato-Katz method and the miracidium hatching technique was considered the diagnostic gold standard. And the diagnostic performance of DDIA was assessed by calculating sensitivity, specificity, positive predictive value (PPV) and negative predictive value (NPV). Sensitivity was calculated as the percentage of participants who were seropositive among those who were positive by stool examination. Specificity was expressed as the percentage of individuals who were seronegative among those who were stool examination negative. PPV was calculated to express the screening efficiency of DDIA by determining the percentage of those who were stool examination positive among those who were seropositive, and NPV was expressed as the percentage of individuals who were stool examination negative among those who were seronegative. 95\% confidence intervals $(\mathrm{CI})$ were calculated for all diagnostic scores. The comparison between groups was analyzed by the Chi-squared test. The relationships between the prevalence and characteristics of DDIA were analyzed by Pearson's correlation analysis. We judged a $P$ value of less than 0.05 to be significant.

For analyzing factors associated with false positive DDIA results, bivariate analysis of various variables was done to identify factors associated with false positivity of DDIA using crude odds ratios (OR) and their 95\% CI. The analysis was restricted to individuals for whom complete sociodemographic and clinical data were available. All variables that were significant based on bivariate analysis $(P<0.05)$ were included in a multivariate model. Adjusted OR and their 95\% CI were calculated. Factors that remained independently associated with the outcome at a $P$ value of $<0.05$ were retained. All analyses were performed using the SPSS (Statistical Products \& Service Solutions) package for windows (SPSS Inc., Chicago, USA, version 13.0).

\section{Results}

\section{Demographic data}

A total of 7,996 people in seven villages voluntarily participated in field survey. $78.60 \%(6285 / 7996)$ of them had completed demographic information and also provided both a single fecal specimen for stool examination and a blood sample for serum for DDIA test. The whole valid population was composed of $48.23 \%$ (3031/6285) men and $51.77 \%$ (3254/6285) women, with a mean age of 38.32 years old (Standard Error (S.E.): 17.71). Most participants were farmers $(62.86 \%, 3951 / 6285)$ and students $(21.70 \%, 1364 / 6285)$.

\section{Endemic areas were characterized with low endemicity of schistosomiasis}

The prevalence of $S$. japonicum infection determined by stool examination was in the range of $0.38 \%-8.23 \%$ in seven communities, while geometric mean EPG ( \pm S.E.) of cases ranged from $5.98 \pm 7.90$ to $45.04 \pm 4.98$ (Table 1). For the whole population, the prevalence of schistosomiasis was $4.20 \%(264 / 6285)$. Among the positive individuals, $84.47 \%(223 / 264)$ were classified as having a low intensity infection (1-99 EPG), 11.74\% (31/ 264) had moderate infections (100-399 EPG), and 3.79\% (10/264) had heavy infections ( $\geq 400$ EPG). 4.44\% (279/ 6285) of the whole population were infected with soiltransmitted helminthes, mainly Ascaris lumbricoides, Trichuris trichiura and hookworm. These data indicated that the study areas were characterized by low prevalence and intensity of schistosome infection. 
Table 1 Prevalence and intensity of S.japonicum infection detected by stool examination

\begin{tabular}{cccccc}
\hline Village & $\begin{array}{c}\text { No. of } \\
\text { examined }\end{array}$ & $\begin{array}{c}\text { No. of } \\
\text { positives }\end{array}$ & $\begin{array}{c}\text { Prevalence } \\
(\%)\end{array}$ & $\begin{array}{c}\text { Geometric mean EPG for infected } \\
\text { (Mean } \pm \text { S.E.) }\end{array}$ & $\begin{array}{c}\text { Other helminthes prevalence rate \% } \\
\text { (No. of positives) }\end{array}$ \\
\hline Longshang & 1044 & 4 & 0.38 & $5.98 \pm 7.90$ & $0.00(0)$ \\
Hebei & 887 & 16 & 1.80 & $10.48 \pm 7.26$ & $0.11(1)$ \\
TieGuai & 859 & 22 & 2.56 & $14.73 \pm 3.18$ & $1.40(12)$ \\
Xinhua & 922 & 46 & 4.99 & $26.05 \pm 6.24$ & $2.93(27)$ \\
Yuye & 824 & 44 & 5.34 & $13.75 \pm 4.21$ & $1.09(9)$ \\
Caohui & 826 & 56 & 6.78 & $22.02 \pm 3.56$ & $20.10(166)$ \\
Jingtou & 923 & 76 & 8.23 & $45.04 \pm 4.98$ & $6.93(64)$ \\
\hline Total & 6285 & 264 & 4.20 & $23.35 \pm 5.05$ & $4.44(279)$ \\
\hline
\end{tabular}

Performance of DDIA compared with stool examination

The results of DDIA compared with those of stool examination are shown in Table 2. Using the results of stool examination as the gold standard, sensitivity, specificity, PPV and NPV of DDIA were calculated.

The sensitivity of DDIA was high with a mean value of $91.29 \%$ (95\% CI: $87.89-94.69 \%$ ) for whole population, with a range of $75.00 \%$ (95\% CI: $32.56-100.00 \%)$ to 95.45\% (95\% CI: 86.75-100.00\%) across the seven villages while no significant difference existed between any two villages $\left(\chi^{2}=2.28, P>0.05\right)$. When infected individuals were divided into infection intensity categories as light, moderate and heavy infection (EPG < 100, 100-399, $\geq 400$, respectively), the sensitivity of DDIA increased with infection intensity, but no significant difference was detected $\left(\chi^{2}=1.29, P>0.05\right)$ (Figure 1A). Among the false negative cases determined by DDIA, $91.30 \%$ (21/ 23) of them had an infection intensity lower than 100 EPG. Sensitivity did not differ significantly between gender and age categories (Figure 1B).

The specificity of DDIA varied significantly from $37.18 \%$ (95\% CI: $33.79-40.57 \%)$ to $78.65 \%$ (95\% CI: 75.92-81.37\%) among seven communities $\left(\chi^{2}=452.87\right.$, $P<0.05)$, with an overall specificity of $53.08 \%$ (95\% CI: $51.82-54.34 \%$ ) (Table 2). Specificity did not vary by gen$\operatorname{der}\left(\chi^{2}=0.83, P>0.05\right)$. However, specificities differed markedly among age groups $\left(\chi^{2}=221.37, P<0.05\right)$, being higher in the youngest and eldest groups but lowest in the age groups corresponding to 26-35 and 36-45 years old (Figure 2).

The PPV of DDIA was very low with the mean value of 7.86\% (95\% CI: 6.91-8.81\%), ranged from 0.52\% (95\% CI: $0-1.10 \%)$ to $18.37 \%$ (95\% CI: $14.48-22.26 \%$ ) in the seven surveyed communities. Pearson's correlation coefficient $(\mathrm{r})$ between the prevalence of schistosome infection and PPV of DDIA was $0.92(P<0.05)$, suggesting that a strong positive correlation exists between the prevalence of schistosome infection and PPV of DDIA at community level. The NPV of DDIA was high in the range of $98.64 \%$ (95\% CI: $97.32-99.96 \%$ ) to $99.85 \%$ (95\% CI: $99.57-$ $100.00 \%$ ) with an average value of $99.29 \%$ (95\% CI: 98.99 99.58\%). A significant negative correlation was detected between the prevalence of schistosome infection and NPV of DDIA with an $r$ value of $-0.88(P<0.05)$.

\section{Factors influencing the false positive DDIA results for schistosome infection}

The results of bivariate logistic regression analysis were listed in Table 3. Individuals aged greater than 15 years old were more likely to have false positive results of DDIA with crude OR in the range of 1.54 (95\% CI: 1.31-1.82) 2.99 (95\% CI: 2.44-3.67), $(P<0.001)$, compared to those

Table 2 Performance characteristics of DDIA compared with stool examination

\begin{tabular}{|c|c|c|c|c|c|c|c|c|}
\hline Criterion assessed & Longshang & Hebei & TieGuai & Xinhua & Yuye & Caohui & Jingtou & Total \\
\hline No. of true positives & 3 & 15 & 21 & 41 & 40 & 51 & 70 & 241 \\
\hline No. of false positives & 579 & 186 & 517 & 383 & 490 & 359 & 311 & 2825 \\
\hline No. of true negatives & 461 & 685 & 320 & 493 & 290 & 411 & 536 & 3196 \\
\hline No. of false negatives & 1 & 1 & 1 & 5 & 4 & 5 & 6 & 23 \\
\hline $\begin{array}{l}\text { Sensitivity } \\
{[\%, 95 \% \mathrm{Cl}]}\end{array}$ & $\begin{array}{c}75.00 \\
{[32.56-100.00]}\end{array}$ & $\begin{array}{c}93.75 \\
{[81.89-100.00]}\end{array}$ & $\begin{array}{c}95.45 \\
{[86.75-100.00]}\end{array}$ & $\begin{array}{c}89.13 \\
{[80.14-98.13]}\end{array}$ & $\begin{array}{c}90.91 \\
{[82.41-99.40]}\end{array}$ & $\begin{array}{c}91.07 \\
{[83.60-98.54]}\end{array}$ & $\begin{array}{c}92.11 \\
{[86.04-98.17]}\end{array}$ & $\begin{array}{c}91.29 \\
{[87.89-94.69]}\end{array}$ \\
\hline $\begin{array}{l}\text { Specificity } \\
{[\%, 95 \% \mathrm{Cl}]}\end{array}$ & $\begin{array}{c}44.33 \\
{[41.31-47.35]}\end{array}$ & $\begin{array}{c}78.65 \\
{[75.92-81.37]}\end{array}$ & $\begin{array}{c}38.23 \\
{[34.94-41.52]}\end{array}$ & $\begin{array}{c}56.28 \\
{[52.99-59.56]}\end{array}$ & $\begin{array}{c}37.18 \\
{[33.79-40.57]}\end{array}$ & $\begin{array}{c}53.38 \\
{[49.85-56.90]}\end{array}$ & $\begin{array}{c}63.28 \\
{[60.04-66.53]}\end{array}$ & $\begin{array}{c}53.08 \\
{[51.82-54.34]}\end{array}$ \\
\hline $\begin{array}{c}\text { PPV } \\
{[\%, 95 \% \mathrm{Cl}]}\end{array}$ & $\begin{array}{c}0.52 \\
[0-1.10]]\end{array}$ & $\begin{array}{c}7.46 \\
{[3.83-11.10]}\end{array}$ & $\begin{array}{c}3.90 \\
{[2.27-5.54]}\end{array}$ & $\begin{array}{c}9.67 \\
{[6.86-12.48]}\end{array}$ & $\begin{array}{c}7.55 \\
{[5.30-9.80]}\end{array}$ & $\begin{array}{c}12.44 \\
{[9.24-15.63]}\end{array}$ & $\begin{array}{c}18.37 \\
{[14.48-22.26]}\end{array}$ & $\begin{array}{c}7.86 \\
{[6.91-8.81]}\end{array}$ \\
\hline $\begin{array}{c}\text { NPV } \\
{[\%, 95 \% \mathrm{Cl}]}\end{array}$ & $\begin{array}{c}99.78 \\
{[99.36-100.00]}\end{array}$ & $\begin{array}{c}99.85 \\
{[99.57-100.00]}\end{array}$ & $\begin{array}{c}99.69 \\
{[99.08-100.00]}\end{array}$ & $\begin{array}{c}99.00 \\
{[98.12-99.87]}\end{array}$ & $\begin{array}{c}98.64 \\
{[97.32-99.96]}\end{array}$ & $\begin{array}{c}98.80 \\
{[97.75-99.85]}\end{array}$ & $\begin{array}{c}98.89 \\
{[98.01-99.77]}\end{array}$ & $\begin{array}{c}99.29 \\
{[98.99-99.58]}\end{array}$ \\
\hline
\end{tabular}

$\mathrm{Cl}$ : Confidence interval 


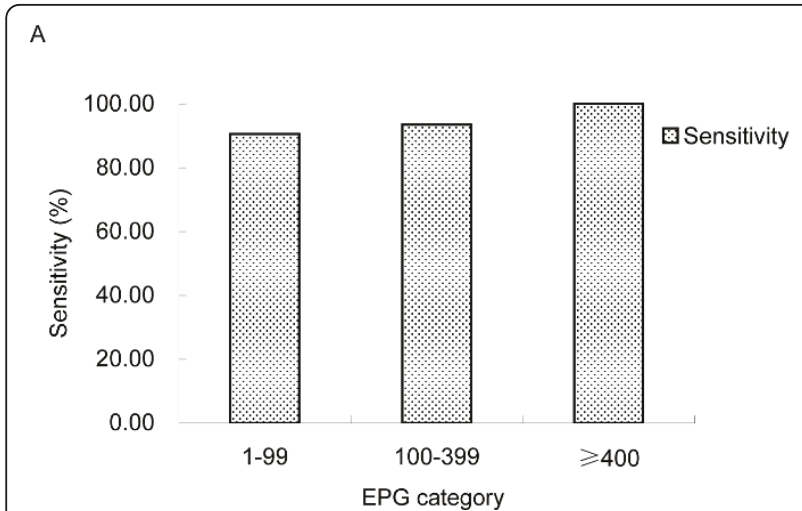

B

Figure 1 Sensitivity of DDIA across (A) infection intensity categories (B) age and gender strata.

individuals aged 6-15 years old. Individuals whose reported occupation was 'fisherman' or 'boatman' (crude OR 2.23, $P<0.001$ ), and students (crude OR $0.52, P<$ 0.001 ) were significantly associated with a false positive DDIA test, as compared with farmers. The history of schistosome infection was strongly associated with false positive DDIA results (crude OR 2.70, $\mathrm{P}<0.001$ ).

In the adjusted multivariate analysis, individuals aged 2635 (adjusted OR: 2.21, 95\% CI: 1.47-3.33) and 36-45 years old (adjusted OR: 1.85, 95\% CI: 1.26-2.74) were at significantly greater risk for a false positive result $(P<0.05)$, compared to the group of 6-15 years old. And fisherman or boatman was a risk factor to cause the false positive results of DDIA with adjusted OR of 1.75 (95\% CI: 1.40-2.17). The false positive rate of DDIA was significantly higher if the individual was infected with schistosomes in the past (OR $=2.47,95 \%$ CI: 2.19-2.79) (Table 3). Sex, education, and infection with soil-transmitted helminthes were not associated with the false positivity of DDIA in either the bivariate or multivariate analysis.

\section{Discussion}

Although vaccination is thought to be an effective supplemental method for control of schistosomiasis, there

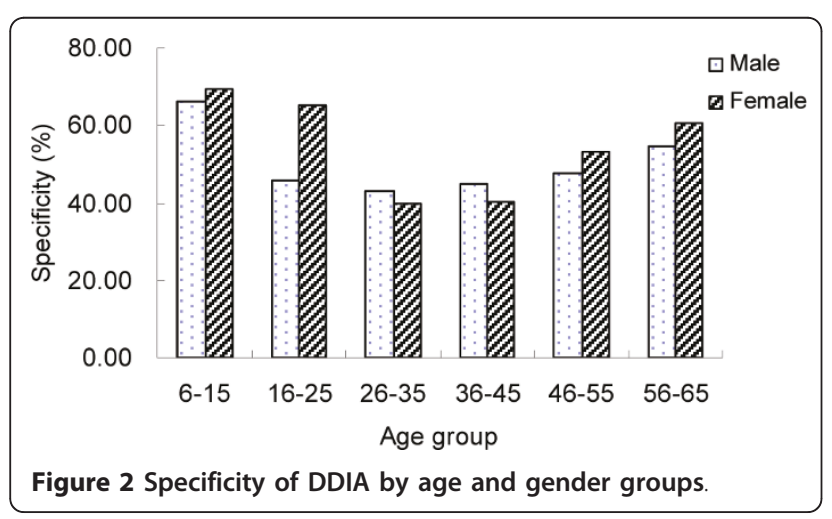

is no effective vaccine available to humans at this point and chemotherapy is still the main strategy for reducing the number of schistosomiasis cases [27-30]. Following the recent significant decreases of prevalence and intensity of schistosome infection in P.R. China, the treatment strategy has been transferred from mass chemotherapy to selective chemotherapy for schistosomiasis control $[2,31,32]$. The infection intensity of patients in our study was very low, and the prevalence of schistosome infection was in the range of $0.38 \%$ to $8.23 \%$. The results proved further that the endemic areas of schistosomiasis japonica in P.R. China are characterized by low prevalence and intensity of schistosome infection, after long-term implementation of national control programs $[13,14]$.

To identify individuals to be targeted for chemotherapy in endemic areas, test-treat is probably the most cost-effective approach [33]. Kato-Katz technique is less sensitive for detection of intestinal schistosomiasis in areas of low endemicity, especially in individuals with low infection intensity $[5,34]$. Immunological assays, with great advantages such as simplicity, rapidity and high sensitivity, have been used to complement stool examinations in P.R. China [13,33]. DDIA is one of few commercially available immunoassays for schistosome infection diagnosis on the market in P.R. China and has been applied in the field for schistosomiasis control based on its quick response and convenience as compared with stool examinations. The practical performance and utility of DDIA in low endemic areas remains unclear. In this study, we evaluated the performance of DDIA, as a primary approach for monitoring the human population in real settings with low endemicity. To evaluate the validity of DDIA, we took the stool examination with the Kato-Katz method and complemented it with the miracidium hatching technique to provide higher sensitivity in parasitological diagnosis of S. japonicum infection in our study $[9,10]$. 
Table 3 Association of demographic and diseases factors with the false positive results of DDIA

\begin{tabular}{|c|c|c|c|c|c|c|}
\hline \multirow[t]{2}{*}{ Variables } & \multicolumn{2}{|c|}{$\begin{array}{l}\text { No. }(\%) \text { of participants with the following DDIA } \\
\text { results }\end{array}$} & \multirow[t]{2}{*}{ Crude OR $(95 \% \mathrm{Cl})$} & \multirow[t]{2}{*}{$P$ value } & \multirow[t]{2}{*}{ Adjusted OR (95\% CI) } & \multirow[t]{2}{*}{$P$ value } \\
\hline & Negative $(n=3196)$ & False positive $(n=2825)$ & & & & \\
\hline \multicolumn{7}{|l|}{ Demographic } \\
\hline \multicolumn{7}{|l|}{ Sex } \\
\hline Female & $1696(53.07)$ & $1500(51.89)$ & 1.00 & 0.36 & 1.00 & 0.27 \\
\hline male & $1466(46.93)$ & $1359(48.11)$ & 1.05(0.95-1.16) & & 1. $07(0.95-1.19)$ & \\
\hline \multicolumn{7}{|l|}{ Age } \\
\hline$\leq 15$ & 875 (27.38) & $420(14.87)$ & 1.00 & & 1.00 & \\
\hline $16-25$ & $186(5.82)$ & $143(5.06)$ & $1.60(1.25-2.05)$ & $<0.001$ & $1.38(0.97-1.97)$ & 0.08 \\
\hline $26-35$ & $230(7.20)$ & $330(11.68)$ & $2.99(2.44-3.67)$ & $<0.001$ & $2.21(1.47-3.33)$ & $<0.001$ \\
\hline $36-45$ & 609 (19.06) & $828(29.31)$ & $2.83(2.42-3.31)$ & $<0.001$ & $1.85(1.26-2.74)$ & $<0.05$ \\
\hline $46-55$ & $616(19.27)$ & $601(21.27)$ & $2.03(1.73-2.39)$ & $<0.001$ & $1.22(0.82-1.82)$ & 0.32 \\
\hline $56-65$ & $680(21.28)$ & $503(17.81)$ & $1.54(1.31-1.82)$ & $<0.001$ & $0.93(0.63-1.38)$ & 0.72 \\
\hline \multicolumn{7}{|l|}{ Occupation } \\
\hline farmer & $1943(60.79)$ & $1874(66.34)$ & 1.00 & & 1.00 & \\
\hline fisherman or boatman & $135(4.22)$ & $290(10.27)$ & $2.23(1.80-2.76)$ & $<0.001$ & $1.75(1.40-2.17)$ & $<0.001$ \\
\hline student & $888(27.78)$ & $442(15.65)$ & $0.52(0.45-0.59)$ & $<0.001$ & $0.97(0.67-1.40)$ & 0.87 \\
\hline others & $230(7.20)$ & $219(7.75)$ & $0.99(0.81-1.20)$ & 0.90 & $0.98(0.79-1.20)$ & 0.82 \\
\hline \multicolumn{7}{|l|}{ Education } \\
\hline illiteracy & $556(17.40)$ & $506(17.91)$ & 1.00 & & 1.00 & \\
\hline$<$ high school & $2454(76.78)$ & $2125(75.22)$ & $0.95(0.83-1.09)$ & 0.47 & $0.98(0.84-1.15)$ & 0.81 \\
\hline high school & $76(2.38)$ & $81(2.87)$ & $1.17(0.84-1.64)$ & 0.36 & $1.05(0.73-1.53)$ & 0.79 \\
\hline college and above & $110(3.44)$ & $113(4.00)$ & $1.13(0.85-1.51)$ & 0.41 & $1.36(0.99-1.86)$ & 0.06 \\
\hline \multicolumn{7}{|l|}{ Disease factors } \\
\hline \multicolumn{7}{|c|}{ History of schistosome infection } \\
\hline No & $1614(50.50)$ & $783(27.72)$ & 1.00 & $<0.001$ & 1.00 & $<0.001$ \\
\hline Yes & $1582(49.50)$ & $2042(72.28)$ & $2.70(2.42-3.01)$ & & $2.47(2.19-2.79)$ & \\
\hline \multicolumn{7}{|c|}{ Other helminthes infection } \\
\hline no & 3053(95.53) & $2711(95.96)$ & 1.00 & 0.40 & 1.00 & 0.50 \\
\hline yes & $143(4.47)$ & $114(4.04)$ & $0.90(0.70-1.15)$ & & $0.91(0.70-1.19)$ & \\
\hline
\end{tabular}

OR : Odds ratio, $95 \% \mathrm{Cl}$ : 95\% confidence intervals

Previous studies conducted in laboratory using wellarchived serum samples and in high endemic areas reported the sensitivities of DDIA ranging from 94.1$100 \%$ for detection of acute or chronic schistosomiasis $[21,22]$. Our study showed that DDIA performed with high sensitivity $(91.29 \%)$ for the whole population in the present areas, although the value reported here is lightly lower than those in previous studies. No significant difference was detected among villages, by age and gender, or even among the subgroups of infection intensity. These data indicated that DDIA is a stable and highly sensitive diagnostic assay. It should be mentioned that $91.30 \%(21 / 23)$ of misdiagnosed cases by DDIA had infection intensities lower than 100 EPG. Thus more sensitive diagnostic assays or methods should be explored to be used in the endemic areas with very low infection intensity where the missed cases will be of great importance for the transmission of schistosomiasis.
The specificity of DDIA was moderate for the whole population (53.08\%), which is much lower than former reports on the same test $[21,22,35]$. We also found the specificities of DDIA varied in communities and across age strata. This could be explained by multiple factors like population background and immune status, the clinical (disease) status and the socio-demographic characteristics of the study participants. Normally the low specificity of an immunological assay can be explained by (1) cross-reactivity with other helminthes, (2) collection of antibodies of past and current exposure, and (3) insensitivity of stool examination $[18,36,37]$. In this study, we proved that the low specificity of DDIA was not caused by cross-reactivity with other helminthes, since only $4.44 \%$ individuals were infected with soil-transmitted helminthes, and no individual was infected with Paragonimus or Clonorchis which have been reported to have high cross-reactivity with schistosomiasis immunodiagnostics [19,23]. Multivariate logistic regression analysis in the present study also supported that 
soil-transmitted helminthes do not influence the specificity of DDIA. The low specificity of DDIA could arguably be due to the well-known insensitivity of single stool KatoKatz thick smears since the communities in this study were characterized with low prevalence and low infection intensity. Increased stool sampling by obtaining more consecutive stools or combined other different diagnostic methods could better define "true" infected cases and increase the specificity of DDIA [5,37]. In our study, multivariate logistic regression analysis showed that the factors significantly associated with the false positive results of DDIA were occupation, age and history of infection. These data support the idea that the antibody-based immunological test provided the indirect proof of past exposure and reflected the history of schistosome infection, which limits its clinical value for confirmation of cases and the success of treatment $[33,37]$.

Predictive values are the main index to assess the yield value of the screening test when it is used in the field [38]. In our study, DDIA had excellent NPV and varied slightly in the range of 98.64 (95\% CI: 97.3299.96\%) - 99.85 (95\% CI: 99.57-100.00\%), although it was significantly associated with the prevalence of the communities. Those data indicated the high probability of truly uninfected individuals among DDIA negatives in the present population. In areas with low prevalence of infection where selective chemotherapy strategy is carried out, if individual tested by DDIA was negative, no further evaluation or treatment needs to be performed. PPV of DDIA was fairly low with a mean value of $7.86 \%$ (95\% CI: $6.91-8.81 \%$ ) and decreased with the prevalence rate determined by stool examination as expected in the low transmission areas. Thus to avoid over-chemotherapy of individuals who have been cured after treatment but still present as antibody positive, a confirmatory method is needed to verify the positive results determined by DDIA [19]. Multiple stool examinations, a combination of different methods, or higher sensitivity and absolute specific molecular methods should be considered to confirm the DDIA positives [5,37-40].

Apart from high sensitivity and specificity, an ideal diagnostic test for use in developing countries should have certain operational characteristics such as being easy to use, rapid and robust, equipment-free and deliverable to end-users [41]. Unlike the CCA-urine dipstick for diagnosing S. mansoni infection, the type of detected specimen of DDIA is serum, which needs to be separated from blood cells through centrifuging or by natural separation by putting blood in low temperature for several hours $[20,42]$. But DDIA meets these criteria to a large extent. The specimen volume needed for DDIA analysis is $20 \mu \mathrm{l}$, which can be obtained through finger or earlobe prick. The test procedure is less than five steps and could be operated without extra equipment except a micropipette. The results can be obtained in 5-10 minutes and read by the naked eye. DDIA is portable and can be stored at room temperature for a long time since former studies reported that the performance of DDIA, including sensitivity, specificity, precision were unchanged at 180 days after being stored at $37^{\circ} \mathrm{C}$ and 360 days after being stored at room temperature [43]. All of these characteristics proved that DDIA could be used in onthe-spot survey in areas of low endemicity. Although the price of DDIA is less than $\$ 1$ per test, further study needs to be done for analyzing the cost-effectiveness of DDIA when used as a screening test in low endemic areas. This will be great of value to decision makers in budget-planning for schistosomiasis control program.

\section{Conclusions}

Our findings revealed that DDIA presented high sensitivity and NPV in low endemicity areas. We also observed that the moderate specificity of DDIA was mainly caused by the insensitivity of stool examination and high exposure history to schistosomes. As a rapid, simple, apparatus-free and sensitive assay, DDIA is of practical and feasible use to be applied as a screening tool in areas of low endemicity for S. japonicum.

\section{Acknowledgements and funding}

Special thanks are addressed to the staffs from provincial institute of Parasitic diseases in Jiangxi, Anhui and Hubei provinces for their efforts during the field and laboratory work. This work was supported by the National S\&T Major Project (Grant No. 2008ZX10004-11) and UNICEF/UNDP/ World BankWHO Special Program (Grant No. A70350)

\section{Author details}

${ }^{1}$ National Institute of Parasitic Diseases, Chinese Center for Disease Control and Prevention, WHO Collaborating Center for Malaria, Schistosomiasis and Filariasis, Shanghai 200025, People's Republic of China. ${ }^{2}$ Provincial Institute of Parasitic Diseases, Nanchang, Jiangxi 330046, People's Republic of China. ${ }^{3}$ Provincial Institute of Parasitic Diseases, Hefei, Anhui 230061, People's Republic of China. ${ }^{4}$ Provincial Institute of Parasitic Diseases, Wuhan, Hubei 430070, People's Republic of China. ${ }^{5}$ London School of Hygiene and Tropical Medicine, London, UK.

Authors' contributions

JX, RWP and XNZ designed the study and participated in data collection. JX and XNZ analyzed the data and wrote the first manuscript. DDL, QZW, LT, TF, XHW and JGG organized the field survey and sample examination. All authors contributed to the manuscript and approved its final version.

\section{Competing interests}

The authors declare that they have no competing interests.

Received: 16 March 2011 Accepted: 20 May 2011

Published: 20 May 2011 


\section{References}

1. Zhou XN, Wang LY, Chen MG, Wu XH, Jiang QW, Chen XY, Zheng J, Utzinger J: The public health significance and control of schistosomiasis in China-then and now. Acta Trop 2005, 96:97-105.

2. Chen XY, Wang LY, Jiming C, Zhou XN, Zheng J, Guo JG, Wu XH, Dirk E, Chen MG: Schistosomiasis control in China: the impact of a 10-year World Bank Loan Project (1992-2001). Bull World Health Organ 2005 83:43-48.

3. Hao $Y$, Zheng $H$, Zhu $R$, Guo JG, Wu XH, Wang LY, Chen Z, Zhou XN: Schistosomiasis status in People's republic of China in 2008. Chin $J$ Schisto Contr 2009, 21:451-456.

4. Zhou XN, Bergquist R, Leonardo L, Yang GJ, Yang K, Sudomo M, Olveda R: Schistosomiasis japonica control and research needs. Adv Parasitol 2010 72:145-178.

5. Lin DD, Liu JX, Liu YM, Hu F, Zhang YY, Xu JM, Li JY, Ji MJ, Bergquist R, Wu GL, et al: Routine Kato-Katz technique underestimates the prevalence of Schistosoma japonicum: a case study in an endemic area of the People's Republic of China. Parasitol Int 2008, 57:281-286.

6. Zhang YY, Luo JP, Liu YM, Wang QZ, Chen JH, Xu MX, Xu JM, Wu J, Tu XM, Wu GL, et al: Evaluation of Kato-Katz examination method in three areas with low-level endemicity of schistosomiasis japonica in China: A Bayesian modeling approach. Acta Trop 2009, 112:16-22.

7. Yu JM, Yuan HC, Chen Q, Yang Q, Zhang SJ, Jiang QW: Comparative study on detection of schistosomiasis infection among repeated Kato-Katz method, IHA and stool hatching test. Chin J schisto Contr 1997, 9:150-153.

8. Yu JM, Yuan HC, Yang QJ, Vlas Sd, Gryseels B: Comparison of Common Methods in the Field Diagnosis of Schistosoma Japonicum Infection. Tongji Da Xue Xue Bao 2001, 22:1-4

9. Xu J, Chen NG, Feng T, Wang EM, Wu XH, Chen HG, Wang TP, Zhou XN, Zheng J: Effectiveness of routinely used assays for the diagnosis of schistosomiasis japonica in the field. Zhongguo Ji Sheng Chong Xue Yu Ji Sheng Chong Bing Za Zhi 2007, 25:175-179.

10. Zhu HQ, Cao CL, Gao FH, Guo JG, Bao ZP, Wang XH, Dang H, Zhou XN: Evaluation of effectiveness of modified Kato-Katz technique for diagnosis of schistosomiasis japonica. Chin J Schisto Contr 2005, 17:273-277.

11. Li JH, Wang TP, Xiao X, Wu WD, Lv DB, Fang GR, Cai W, Zheng J, Xu J, Wang RR: Cost-effectiveness analysis on different schistosomiasis case screen methods in hypo-endemic area. Shi Yong Ji Sheng Chong Bing Za Zhi 2002, 10:145-147.

12. Mao LX, Zheng JJ, Chen JH, Shao GH, Wen LY, Wang XM: Investigation on compliance of schistosomiasis examination in Jiangshan City. Chin J Schisto Contr 2008, 20:125-129.

13. Zhu YC: Immunodiagnosis and its role in schistosomiasis control in China: a review. Acta Trop 2005, 96:130-136.

14. Zhou XN, Guo JG, Wu XH, Jiang QW, Zheng J, Dang H, Wang XH, Xu J, Zhu HQ, Wu GL, et al: Epidemiology of schistosomiasis in the People's Republic of China, 2004. Emerg Infect Dis 2007, 13:1470-1476.

15. Zhu R, Dang H, Zhang YJ, Li HZ, Zheng CJ, Wu XH, Guo JG: National surveillance of schistosomiasis in China,2005-2008. Chin J Schisto Contr 2009, 21:358-362.

16. Wu XH, Xu J, Li SZ, Huang YX, Wang TP, Wu CG, Huang XB, Miu F, Chen Z, Wang LY: Prediction and surveillance of schistosomiais japonica in potential endemic areas in China, 2008. Chin J Schisto Contr 2009, 21:353-357.

17. Zhou YB, Yang MX, Wang QZ, Zhao GM, Wei JG, Peng WX, Jiang QW: Field comparison of immunodiagnostic and parasitological techniques for the detection of Schistosomiasis japonica in the People's Republic of China. Am J Trop Med Hyg 2007, 76:1138-1143.

18. Lin DD, Xu JM, Zhang YY, Liu YM, Hu F, Xu XL, Li JY, Gao ZL, Wu HW, Kurtis J, et al: Evaluation of IgG-ELISA for the diagnosis of Schistosoma japonicum in a high prevalence, low intensity endemic area of China. Acta Trop 2008, 107:128-133.

19. Xu J, Peeling RW, Chen JX, Wu XH, Wu ZD, Wang SP, Feng T, Chen SH, $\mathrm{Li} \mathrm{H}$, Guo JG, et al: Evaluation of nine immunoassays for the diagnosis of schistosoma japnicum Infection using archived sera. PLOS Negl Trop Dis 2011, 5:e949.

20. Standley C, Lwambo N, Lange C, Kariuki H, Adriko M, Stothard J: Performance of circulating cathodic antigen (CCA) urine-dipsticks for rapid detection of intestinal schistosomiasis in schoolchildren from shoreline communities of Lake Victoria. Parasit Vectors 2010, 3:7.

21. Zhu YC, He W, Liang YS, Xu M, Yu CX, Hua WQ, Chao GQ: Development of a rapid, simple dipstick dye immunoassay for schistosomiasis diagnosis. $J$ immunol methods 2002, 266:1-5.

22. Zhu YC, He W, Dai JR, Xu M, Liang YS, Tang JX, Hua WQ, Cao GQ, Chen HG, Lou PA: Application of dipstick dye immunoassay (DDIA) kit on detection of schistosomiasis japonica on large scale in endemic areas. Chin J schisto Contr 2005, 17:246-249.

23. Xu J, Feng T, Guo JG, Zheng H, Wang Q, Wu XH, Zhou XN: Comprehensive evaluation of several diagnosis agents of schistosomiasis japonica in China. Chin J Schisto Contr 2005, 17:116-119.

24. Katz N, Chaves A, Pellegrino J: A simple device for quantitative stool thick-smear technique in schistosomiasis mansoni. Rev Inst Med Trop Sao Paulo 1972, 14:397-400

25. Department of Diseases Control: Textbook for schistosomiasis control. Shanghai Shanghai Publishing House for Science and Technology i, 32000

26. WHO: Prevention and control of schistosomiasis and soil-transmitted helminthiasis:report of a WHO expert committee. In WHO Tech Rep Ser 912. Volume 912. Geneva; 2002:2-5.

27. Wang X, Zhang L, Chi Y, Hoellwarth J, Zhou S, Wen X, He L, Liu F, Wu C, Su C: The nature and combination of subunits used in epitope-based Schistosoma japonicum vaccine formulations affect their efficacy. Parasit Vectors 3:109.

28. Bergquist $\mathrm{R}$, Utzinger J, McManus DP: Trick or treat: the role of vaccines in integrated schistosomiasis control. PLoS Negl Trop Dis 2008, 2:e244.

29. Bergquist NR, Leonardo LR, Mitchell GF: Vaccine-linked chemotherapy: can schistosomiasis control benefit from an integrated approach? Trends Parasitol 2005, 21:112-117.

30. Zhu R, Gray DJ, Thrift AP, Williams GM, Zhang Y, Qiu DC, Zheng F, Li YS, Guo J, Zhu HQ, et al: A 5-year longitudinal study of schistosomiasis transmission in Shian village, the Anning River Valley, Sichuan Province, the Peoples' Republic of China. Parasit Vectors 4:43.

31. Chen MG: Use of praziquantel for clinical treatment and morbidity control of schistosomiasis japonica in China: a review of 30 years' experience. Acta Trop 2005, 96:168-176.

32. Lin DD, Hu GH, Zhang SJ: Optimal combined approaches of field intervention for schistosomiasis control in China. Acta Trop 2005, 96:242-247.

33. Wu GL: A historical perspective on the immunodiagnosis of schistosomiasis in China. Acta Trop 2002, 82:193-198.

34. Enk MJ, Lima AC, Massara CL, Coelho PM, Schall VT: A combined strategy to improve the control of Schistosoma mansoni in areas of low prevalence in Brazil. Am J Trop Med Hyg 2008, 78:140-146.

35. Liang YS, Zhu YC, Dai JR, He W, Xu M, Li YL, Wang SW, Tang JX, Hua WQ $\mathrm{Li} \mathrm{HJ}$, et al: Field application of dipstick dye immunoassay kit for detecting schistosomiasis in mountainous endemic regions in Yunnan Province. Chin J Schisto Contr 2005, 17:405-408.

36. Xiao X, Wang T, Ye H, Qiang G, Wei H, Tian Z: Field evaluation of a rapid, visually-read colloidal dye immunofiltration assay for Schistosoma japonicum for screening in areas of low transmission. Bull World Health Organ 2005, 83:526-533.

37. Bergquist $R$, Johansen MV, Utzinger J: Diagnostic dilemmas in helminthology: what tools to use and when? Trends Parasitol 2009, 25:151-156.

38. Banoo S, Bell D, Bossuyt P, Herring A, Mabey D, Poole F, Smith PG, Sriram N, Wongsrichanalai C, Linke R, et al: Evaluation of diagnostic tests for infectious diseases: general principles. Nat Rev Microbiol 2006, 4 : S20-32.

39. Xu J, Rong $R$, Zhang $H Q$, Shi CJ, Zhu XQ, Xia CM: Sensitive and rapid detection of Schistosoma japonicum DNA by loop-mediated isothermal amplification (LAMP). Int J Parasitol 2010, 40:327-331.

40. Lier T, Simonsen GS, Wang T, Lu D, Haukland HH, Vennervald BJ, Hegstad J, Johansen MV: Real-time polymerase chain reaction for detection of lowintensity Schistosoma japonicum infections in China. Am J Trop Med Hyg 2009, 81:428-432.

41. Mabey D, Peeling RW, Ustianowski A, Perkins MD: Diagnostics for the developing world. Nat Rev Microbiol 2004, 2:231-240. 
42. Standley CJ, Adriko M, Arinaitwe M, Atuhaire A, Kazibwe F, Fenwick A, Kabatereine NB, Stothard JR: Epidemiology and control of intestinal schistosomiasis on the Sesse Islands, Uganda: integrating malacology and parasitology to tailor local treatment recommendations. Parasit Vectors 2010, 3:64.

43. He W, Zhu YC, Cao GQ, Xin XF: Study on stability of dipstick dye immunoassay kit for schistosomiasis diagnosis. Chin J Schisto Contr 2005, 17:262-264.

doi:10.1186/1756-3305-4-87

Cite this article as: Xu et al.: Performance of a dipstick dye

immunoassay for rapid screening of Schistosoma japonicum infection in areas of low endemicity. Parasites \& Vectors 2011 4:87.

Submit your next manuscript to BioMed Central and take full advantage of:

- Convenient online submission

- Thorough peer review

- No space constraints or color figure charges

- Immediate publication on acceptance

- Inclusion in PubMed, CAS, Scopus and Google Scholar

- Research which is freely available for redistribution

Submit your manuscript at www.biomedcentral.com/submit 